\title{
Interstitial pneumonia following administration of pegfilgrastim during carboplatin and etoposide chemotherapy for small-cell lung cancer
}

\author{
MASAYUKI SHIRASAWA ${ }^{1}$, YOSHIRO NAKAHARA ${ }^{1}$, HIDEYUKI NIWA $^{1}$, SHINYA HARADA ${ }^{1}$, \\ TAKAHIRO OZAWA ${ }^{1}$, SEIICHIRO KUSUHARA ${ }^{1}$, MASASHI KASAJIMA ${ }^{1}$, \\ YASUHIRO HIYOSHI ${ }^{1}$, JIICHIRO SASAKI ${ }^{2}$ and NORIYUKI MASUDA ${ }^{1}$ \\ ${ }^{1}$ Department of Respiratory Medicine; ${ }^{2}$ Research and Development Center for New Medical Frontiers, \\ Kitasato University School of Medicine, Sagamihara, Kanagawa 252-0374, Japan
}

Received June 28, 2016; Accepted August 18, 2016

DOI: $10.3892 /$ mco.2016.1062

\begin{abstract}
Pegfilgrastim is a long-acting granulocyte colony-stimulating factor formulation that has been approved for the prevention of febrile neutropenia. We herein report a case of interstitial pneumonia following administration of pegfilgrastim. A 65-year-old man with stage IV small-cell lung cancer was treated with carboplatin and etoposide as third-line chemotherapy. Pegfilgrastim was administered during the second cycle of chemotherapy. On the day after the administration of pegfilgrastim, interstitial pneumonia developed. The respiratory condition improved with pulse steroid therapy; however, the patient eventually succumbed to cancer progression. In conclusion, interstitial pneumonia due to pegfilgrastim is rare; however, physicians should be aware of the possibility of this adverse effect.
\end{abstract}

\section{Introduction}

Febrile neutropenia is a life-threatening condition characterized by fever in addition to chemotherapy-induced neutropenia. The longer the duration of neutropenia, the higher the risk of developing febrile neutropenia.

Therefore, granulocyte-colony stimulating factor (G-CSF) is often used to manage chemotherapy-induced neutropenia. Furthermore, prophylactic administration of G-CSF is recommended for patients at high risk of febrile neutropenia by several guidelines $(1,2)$.

Correspondence to: Dr Yoshiro Nakahara, Department of Respiratory Medicine, Kitasato University School of Medicine, 1-15-1 Kitasato, Minami-ku, Sagamihara, Kanagawa 252-0374, Japan

E-mail: md100062@kitasato-u.ac.jp

Key words: pegfilgrastim, interstitial lung disease, lung cancer
Pegfilgrastim is a long-acting granulocyte-colony stimulating factor (G-CSF) formulation. A phase III randomized trial demonstrated that pegfilgrastim significantly reduced the incidence of febrile neutropenia in breast cancer patients (3). According to the data, pegfilgrastim has been approved in Japan since 2014 and several associated adverse effects, including rash, joint pain, diarrhea and fever, have been reported. However, interstitial lung disease (ILD) during pegfilgrastim treatment has rarely been reported. We herein present a case of ILD following administration of pegfilgrastim.

\section{Case report}

An asymptomatic 64-year-old man was referred to another hospital due to abnormal nodular shadows in the left upper lung on chest computed tomography (CT) scan. The smoking history was 44 pack-years. Thoracoscopic pleural biopsy was performed and the patient was diagnosed with small-cell lung cancer (SCLC) stage IV (T4N2M1a). The patient was treated every 4 weeks with four cycles of first-line chemotherapy, including cisplatin $\left(60 \mathrm{mg} / \mathrm{m}^{2}\right)$ and irinotecan $\left(60 \mathrm{mg} / \mathrm{m}^{2}\right)$ on day 1 and irinotecan $\left(60 \mathrm{mg} / \mathrm{m}^{2}\right)$ alone on day 8 . After the first-line chemotherapy, complete response (CR) was observed.

However, 1 month after the fourth cycle of chemotherapy, the infiltrative shadows progressed. SCLC progression was hypothesized and the patient was treated with amrubicin monotherapy (40 mg/m $\mathrm{m}^{2}$ on days 1,2 and 3) as second-line chemotherapy every 3 weeks. Due to certain circumstances of the patient, he was transferred to our hospital.

After the fourth cycle of chemotherapy, the SCLC continued to progress. The patient received combination chemotherapy with carboplatin (area under the curve=5) and etoposide ( $80 \mathrm{mg} / \mathrm{m}^{2}$ on days 1,2 and 3 ) as third-line chemotherapy every 3 weeks. The adverse events of the first course were grade 4 neutropenia and grade 3 thrombocytopenia. Prior to the second cycle of chemotherapy, the performance status (PS) of the patient was 3. However, we decided to administer a second cycle of chemotherapy, 


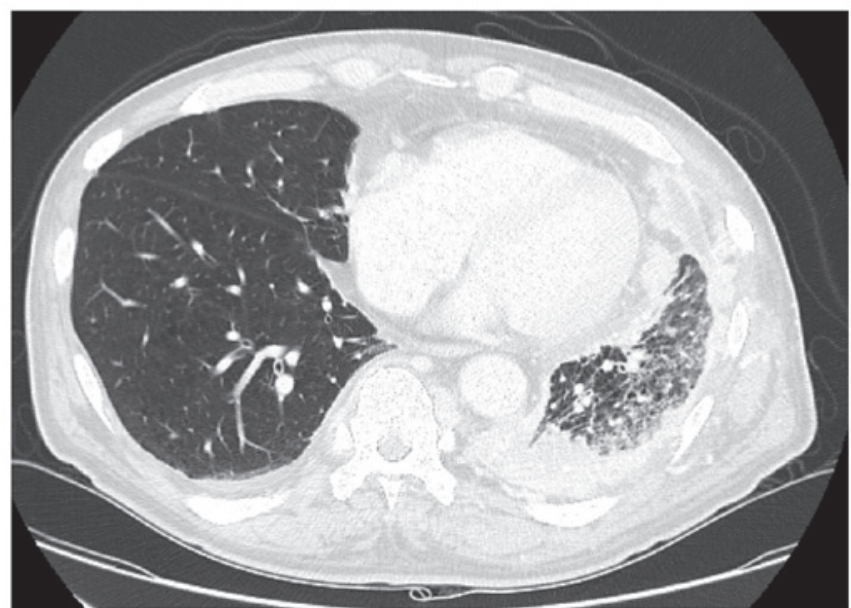

Figure 1. Chest computed tomography scan on admission showing cancer of the left lung.

as this combination therapy was considered to be effective (Fig. 1). Therefore, pegfilgrastim was added to prevent febrile neutropenia.

One day after pegfilgrastim administration, the patient experienced sudden deterioration of his respiratory status. On physical examination there were fine crackles on the right side of the chest, and the CT scan revealed diffuse infiltrative shadows (Fig. 2). Subsequent tests for infectious diseases, such as sputum, blood and urine cultures, were all normal. From these results, we considered that the patient's clinical course was due to drug-induced lung injury. The patient was treated with methylprednisolone (1,000 mg/day for 3 days). The interstitial pneumonia improved after 10 days of pulse steroid therapy (Fig. 3). However, the patient succumbed to cancer progression 1 month after the occurrence of interstitial pneumonia.

\section{Discussion}

Our patient was administered pegfilgrastim for the prevention of febrile neutropenia, but ILD developed on the next day. Apart from pegfilgrastim, carboplatin and etoposide were suspected as the other possible offending drugs. The drug-induced lymphocyte stimulation test for these three drugs was negative. ILD secondary to carboplatin and etoposide is extremely rare (4). Moreover, the disease did not manifest in the patient after the first course of combination chemotherapy with carboplatin and etoposide. Therefore, in this case, we considered that the drug most likely responsible for ILD was pegfilgrastim.

To the best of our knowledge, interstitial pneumonia has not been reported in clinical trials on pegfilgrastim $(3,5-8)$. However, several reports indicated that the administration of G-CSF may be associated with lung injury. Matthews reported that pneumotoxicity occurred in 3 out of 5 patients with Hodgkin's lymphoma who received G-CSF with doxorubicin, bleomycin, vinblastine and dacarbazin (ABVD) therapy (9). Yokose et al reported that pulmonary toxicity had occurred in 6 out of 52 patients with non-Hodgkin lymphoma who received G-CSF with cyclophosphamide, doxorubicin,

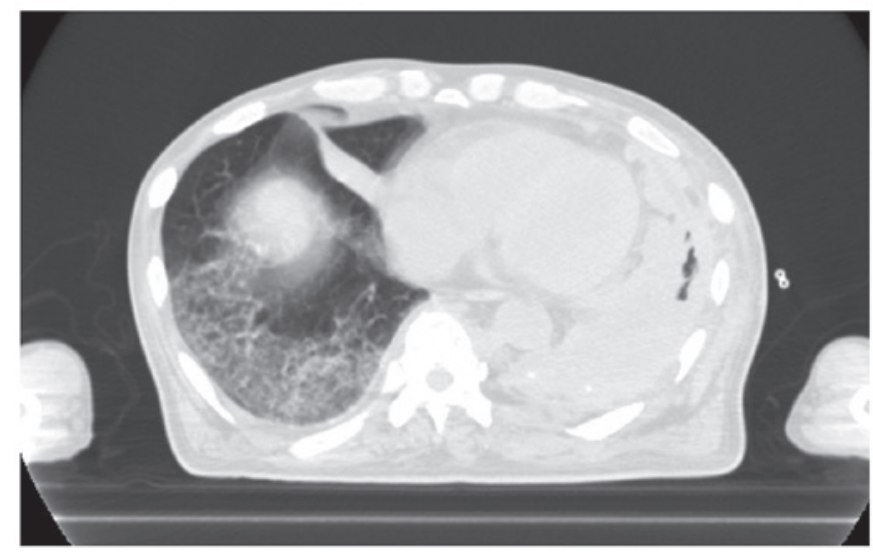

Figure 2. Chest computed tomography scan showing a diffuse infiltrative shadow.

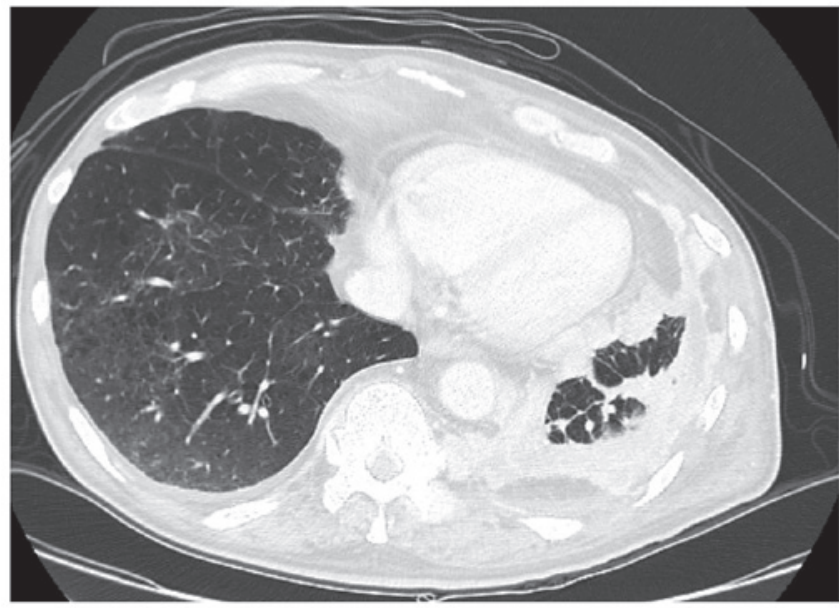

Figure 3. Chest computed tomography scan showing improvement of the interstitial pneumonia.

vincristine and prednisolone (CHOP) therapy (10). The study demonstrated that the mean peak leukocyte count with each therapy cycle had been associated with development of pulmonary toxicity, and concluded that lowering the G-CSF dose appeared to be useful in the prevention of this toxicity. Furthermore, Ruiz-Argüelles et al reported a case of pulmonary toxicity after the administration of G-CSF without any chemotherapy (11).

Adachi et al reported that the mechanism of ILD due to G-CSF was enhancement of the infiltration of the alveoli by alkaline phosphatase-positive neutrophils (12). However, this mechanism has not been fully elucidated.

Smoking history and poor PS were reported to be risk factors of drug-induced lung injury (13). Furthermore, Niitsu et al reviewed 20 cases of interstitial pneumonia secondary to treatment with G-CSF, and reported that it occurred predominantly in patients aged $\geq 60$ years (14).

Our patient was 64 years old, had a history of heavy smoking and his PS was 3 at the start of pegfilgrastim administration, placing him at risk to develop ILD.

In conclusion, drug-induced lung injury by pegfilgrastim is rare. However, physicians should be aware of the possibility of this adverse effect. 


\section{References}

1. Smith TJ, Khatcheressian J, Lyman GH, Ozer H, Armitage JO, Balducci L, Bennett CL, Cantor SB, Crawford J, Cross SJ, et al: 2006 update of recommendations for the use of white blood cell growth factors: An evidence-based clinical practice guideline. J Clin Oncol 24: 3187-3205, 2006.

2. Aapro MS, Bohlius J, Cameron DA, Dal Lago L, Donnelly JP, Kearney N, Lyman GH, Pettengell R, Tjan-Heijnen VC, Walewski J, et al: 2010 update of EORTC guidelines for the use of granulocyte-colony stimulating factor to reduce the incidence of chemotherapy-induced febrile neutropenia in adult patients with lymphoproliferative disorders and solid tumours. Eur J Cancer 47: 8-32, 2011

3. Kosaka Y, Rai Y, Masuda N, Takano T, Saeki T, Nakamura S, Shimazaki R, Ito Y, Tokuda Y and Tamura K: Phase III placebo-controlled, double-blind, randomized trial of pegfilgrastim to reduce the risk of febrile neutropenia in breast cancer patients receiving docetaxel/cyclophosphamide chemotherapy. Support Care Cancer 23: 1137-1143, 2015.

4. Okamoto H, Watanabe K, Kunikane H, Yokoyama A, Kudoh S, Asakawa T, Shibata T, Kunitoh H, Tamura T and Saijo N: Randomised phase III trial of carboplatin plus etoposide vs split doses of cisplatin plus etoposide in elderly or poor-risk patients with extensive disease small-cell lung cancer: JCOG 9702. Br J Cancer 97: 162-169, 2007.

5. Yamamoto N, Sekine I, Nakagawa K, Takada M, Fukuoka M, Tanigawara Y and Saijo N: A pharmacokinetic and dose escalation study of pegfilgrastim (KRN125) in lung cancer patients with chemotherapy-induced neutropenia. Jpn J Clin Oncol 39: 425-430, 2009

6. Lee KH, Kim JY, Lee MH, Han HS, Lim JH, Park KU, Park IH, Cho EK, Yoon SY, Kim JH, et al: A randomized, multicenter, phase II/III study to determine the optimal dose and to evaluate the efficacy and safety of pegteograstim (GCPGC) on chemotherapy-induced neutropenia compared to pegfilgrastim in breast cancer patients: KCSG PC10-09. Support Care Cancer 24: 1709-1717, 2016
7. Masuda N, Tokuda Y, Nakamura S, Shimazaki R, Ito Y and Tamura K: Dose response of pegfilgrastim in Japanese breast cancer patients receiving six cycles of docetaxel, doxorubicin, and cyclophosphamide therapy: A randomized controlled trial. Support Care Cancer 23: 2891-2898. 2015.

8. Yang BB, Morrow PK, Wu X, Moxness M and Padhi D: Comparison of pharmacokinetics and safety of pegfilgrastim administered by two delivery methods: On-body injector and manual injection with a prefilled syringe. Cancer Chemother Pharmacol 75: 1199-1206, 2015.

9. Matthews JH: Pulmonary toxicity of ABVD chemotherapy and G-CSF in Hodgkin's disease: Possible synergy. Lancet 342: 988 , 1993.

10. Yokose N, Ogata K, Tamura H, An E, Nakamura K, Kamikubo K, Kudoh S, Dan K and Nomura T: Pulmonary toxicity after granulocyte colony-stimulating factor-combined chemotherapy for non-Hodgkin's lymphoma. Br J Cancer 77: 2286-2290, 1998.

11. Ruiz-Argüelles GJ, Arizpe-Bravo D, Sánchez-Sosa S, Rojas-Ortega S, Moreno-Ford V and Ruiz-Argüelles A: Fatal G-CSF-induced pulmonary toxicity. Am J Hematol 60: 82-83, 1999.

12. Adachi K, Suzuki M, Sugimoto T, Yorozu K, Takai H, Uetsuka K, Nakayama $\mathrm{H}$ and Doi K: Effects of granulocyte colony-stimulating factor (G-CSF) on bleomycin-induced lung injury of varying severity. Toxicol Pathol 31: 665-673, 2003.

13. Kudoh S, Kato H, Nishiwaki Y, Fukuoka M, Nakata K, Ichinose Y, Tsuboi M, Yokota S, Nakagawa K, Suga M, et al: Interstitial lung disease in Japanese patients with lung cancer: A cohort and nested case-control study. Am J Respir Crit Care Med 177: 1348-1357, 2008

14. Niitsu N, Iki S, Muroi K, Motomura S, Murakami M, Takeyama H, Ohsaka A and Urabe A: Interstitial pneumonia in patients receiving granulocyte colony-stimulating factor during chemotherapy: Survey in Japan 1991-96. Br J Cancer 76: 1661-1666, 1997. 